\title{
Evaluation of a Direct Agglutination Test for Detection of Antibodies Against Toxoplasma gondii in Cat, Pig and Sheep Sera
}

The protozoan parasite Toxoplasma gondii is the causative agent of the zoonosis toxoplasmosis. In sheep and goats, it is one of the most prevalent causes of infectious abortion. Also in pregnant women, a primary infection can result in miscarriage. Humans acquire the infection either by ingestion of oocysts excreted by cats, the definitive host of the parasite, or by eating raw or undercooked meat from latently infected animals (Dubey \& Beattie 1988). In Sweden, toxoplasmosis is a notifiable disease, and cases of clinical disease in humans as well as animals must be reported. In both veterinary and human medicine serological assays based on detecting the humoral antibody response of the host against the parasite are used as diagnostic tools. So far, solid phase assays, such as the indirect fluorescent antibody test (IFAT) and the enzyme-linked immunosorbent assay (ELISA), have been widely used to diagnose $T$. gondii infection in many species including cats, pigs and sheep (Dubey \& Beattie 1988). However, both IFAT and ELISA require appropriate anti-species specific immunoglobulins (Ig) that must be carefully evaluated for each species prior to use. This makes these assays complicated and time consuming. Consequently, alternative, simpler methods that do not require specific antisera would be of great value. The direct agglutination test (DA), which is based on the principle that formalin-treated organisms ag- glutinate in the presence of specific IgG antibodies, is such an assay (Fulton \& Turk 1959). The DA-test is widely used in human medicine as a screening test for $T$. gondii infection but it has not yet been thoroughly evaluated for use in veterinary medicine (Uggla \& Buxton 1990).

In the present study, the applicability of a commercially available DA-test (ToxoScreen DA; bioMérieux, Marcy-l'Etoile, France) was evaluated for use in cats, pigs and sheep. Its sensitivity and specificity was evaluated by comparison with the IFAT, and its feasibility as a laboratory method was considered.

In total, 60 feline, 60 porcine and 58 ovine sera were analysed both by Toxo-Screen DA and by the IFAT. The majority of the sera were obtained from field cases, while 15 porcine sera were from specific disease free (SPF) pigs and 15 ovine sera were from sheep experimentally inoculated with $T$. gondii oocysts. Thirty sera from each species had previously been deemed positive by IFAT or ELISA. In addition, positive and negative species specific control sera were used in both the DA-test and the IFAT.

The IFAT-test used in this study has been described in detail earlier (Uggla \& Hjort 1984). Briefly, T. gondii tachyzoites smeared on microscopic slides (Toxospot IF; bioMérieux) were incubated for $30 \mathrm{~min}$ with sera serially 
Table 1. Comparison between direct agglutionation test (DA) and indırect fluorescent antıbody test (IFAT) for detection of antibodies against $T$ gondll in cat, pig and sheep sera.

\begin{tabular}{|c|c|c|c|c|c|c|}
\hline \multirow{2}{*}{ Sera } & & & \multicolumn{2}{|c|}{ IFAT } & \multirow{2}{*}{$\begin{array}{l}\text { Sensi- } \\
\text { tivity }\end{array}$} & \multirow{2}{*}{$\begin{array}{l}\text { Spect } \\
\text { ficty }\end{array}$} \\
\hline & & & positive & negatıve & & \\
\hline \multirow{2}{*}{$\begin{array}{l}\text { Cat } \\
(n=60)\end{array}$} & DA & positive & 27 & 1 & & \\
\hline & & negative & 1 & 31 & 0.96 & 0.97 \\
\hline \multirow{2}{*}{$\begin{array}{l}\text { Pig } \\
(n=60)\end{array}$} & DA & positive & 30 & 0 & & \\
\hline & & negative & 0 & 30 & 1.00 & 1.00 \\
\hline \multirow{2}{*}{$\begin{array}{l}\text { Sheep } \\
(n=58)\end{array}$} & DA & positive & 31 & 0 & & \\
\hline & & negatıve & 0 & 27 & 1.00 & 1.00 \\
\hline
\end{tabular}

diluted in twofold steps starting at 1:40. After washing twice in phosphate buffered saline, pH 7.2 (PBS), antibodies were localised depending on the source of the serum either with: (1) fluorescein (FITC)-labelled rabbit anti-cat Ig (Organon Teknika Int., Turnhout, Belgium); (2) FITC-labelled rabbit anti-pig Ig (Dakopatts, Copenhagen, Denmark); or (3) FITC-labelled rabbit anti-sheep Ig (Dakopatts). Subsequently, cover glasses were mounted with buffered glycerine and the preparations read under an oil immersion lens at a magnification of $400 \mathrm{x}$ in a fluorescence microscope (Leitz Orthoplan, Germany). An unbroken peripheral line of bright fluorescence around the organisms was considered as a positive reaction. Sera were deemed positive if a positive reaction was observed when tested at dilution 1:40. The value of the titre was determined as the highest dilution giving a positive reaction.

The DA-test was performed in accordance with the instructions of the manufacturer. Briefly, all sera were analysed in duplicates in U-shaped microtitre wells. Prior to application, sera were diluted 1:20 and 1:2000 in PBS, and $25 \mu \mathrm{l}$ of the diluted sera were added to each well. Two wells on each plate were filled with $25 \mu \mathrm{l}$ PBS and served as antigen controls. In addition, wells on each plate were filled with control sera; 2 each with the purchased positive and negative control goat sera and 2 each with positive and negative species specific control sera from our own sources. Then, in order to reduce non-specific agglutination (Desmonts \& Remington 1980), $25 \mu 10.2$ M 2mercaptoethanol (2ME) in PBS was added to each of the 96 wells of the plates giving a final serum dilution of $1: 40$ and $1: 4000$, respectively. Finally, $50 \mu \mathrm{l}$ of the antigen suspension diluted 1:5 in boric acid buffered saline, $\mathrm{pH}$ 8.95 (BABS), was added per well. The plates were covered with self-adhesive sheets, placed on a shaker for $1 \mathrm{~min}$ and then incubated for $5 \mathrm{~h}$ at room temperature $\left(18-25^{\circ} \mathrm{C}\right)$ before reading. If required, a second reading was done after $18 \mathrm{~h}$ of incubation. A sample was regarded as positive when the antigen agglutinated in a mat covering about half of the well base. In order to quantify the serum titre, the 1:20 and 1:2000 serum dilutions were serially diluted by three-fold steps and analysed as described above. The value of the titre was determined as the highest dilution giving a positive reaction.

As seen in Table 1, 176 (99\%) of the 178 sera analysed showed equal qualitative results in both tests (positive or negative). Among the feline sera one serum proved negative in the IFAT but positive in the DA, and one serum 


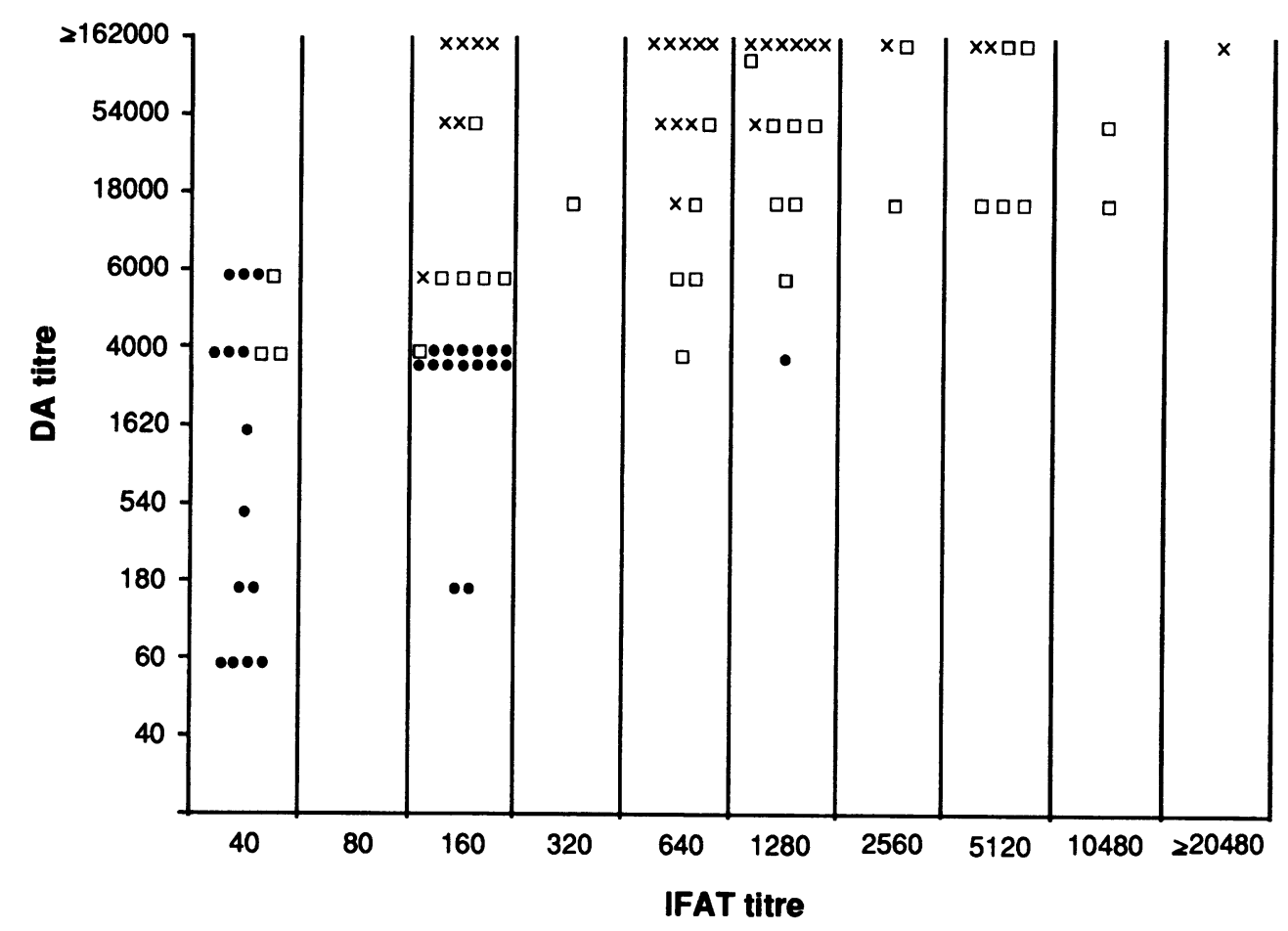

Figure 1. Comparison of antibody titres against $T$. gondil detected by direct agglutionation test (DA) and indirect fluorescent antibody test (IFAT), in 27 cat $(\times), 30 \mathrm{plg}(\bullet)$ and 31 sheep ( $\square$ ) sera.

showed the opposite discrepancy. However, the Toxo-Screen DA titres were in general higher than the titres scored by the IFAT (Fig. 1).

The present study clearly shows that when compared to IFAT, the Toxo-Screen DA is a sensitive and specific assay for the detection of antibodies to T. gondii in sera from cat, pig and sheep. There were only 2 exceptions to the excellent agreement between the qualitative results obtained by the 2 assays. The reason why 1 serum proved negative in the Toxo-Screen DA but positive in the IFAT may have been that this serum was collected at an early stage of infection and thus contained IgM antibodies only. Addition of $2 \mathrm{ME}$ in the Toxo-Screen DA probably eliminates both non-specific agglutination of "normal" IgM and agglutionation of $T$. gondii specific IgM (Desmonts \& Remington 1980). In the other case, with a positive Toxo-Screen DA (titre 1:40) and a negative IFAT result, the explanation for this outcome could be either that the Toxo-Screen DA detects an extremely low level of antibodies or that the DA-test showed a false positive result.

This study also shows that the Toxo-Screen DA method has several advantages in veterinary routine diagnostics. As this test does not require anti-species specific immunoglobulin preparations the same procedure can be used for all kinds of sera. The reliability of the test is checked by control sera as well as an antigen control. Furthermore, the DA-assay is 
rapid and easy to perform and does not require any sophisticated equipment.

Britt-Loulse Ljungstrom, Anna Lundén, Johan Hoglund \& Goran Zakrisson

Department of Parasitology, Swedish University of Agricultural Sciences and Natıonal Veterinary Inst1tute, Uppsala Sweden.

\section{References}

Desmonts G, Remington JS - Direct agglutination test for diagnosis of Toxoplasma infection:
Method for increasing sensitivity and specificity. J. clin. Microbiol. 1980, 11, 562-568.

Dubey JP, Beattle CP: Toxoplasmosis of anımals and man. CRC Press, Boca Raton, Florida, USA, 1988.

Fulton JD, Turk $J L$ : Direct agglutınation test for Toxoplasma gondii. Lancet 1959, 2, 1068-1069.

Uggla A, Buxton D: Immune responses agınst Toxoplasma and Sarcocystıs infections in ruminants: diagnosis and prospects for vaccination. Rev Scr. Tech. Off. Int. Epız. 1990, 9, 441-462.

Uggla A, HJort M. A serological study on the prevalence of Toxoplasma gondil in meat producing animals in Sweden. Acta vet. scand. 1984, 25, 567-576.

(Recelved December 21, 1993; accepted March 30, 1994)

Reprints may be requested from. Department of Parasitology, Swedish Unıversity of Agricultural Sciences and National Veterınary Institute, P.O. Box 7073, S-750 07, Uppsala Sweden. 

